\title{
A SHIELD-DATA-BASED HORIZON CONTROL APPROACH FOR THIN SEAM COAL MINING UTILIZING PLOW TECHNOLOGY
}

\author{
*S. Beitler ${ }^{1}$ and M. Holm ${ }^{1}$, T. Arndt ${ }^{1}$, A. Mozar ${ }^{2}$, M. Junker ${ }^{2}$, C. Bohn ${ }^{1}$ \\ ${ }^{1}$ Clausthal University of Technology \\ Institute of Electric Information Technology \\ Leibnizstraße 28 \\ 38678 Clausthal-Zellerfeld, Germany \\ (*Corresponding author: beitler@iei.tu-clausthal.de) \\ ${ }^{2} R A G$ Deutsche Steinkohle \\ Shamrockring 1 \\ 44623 Herne, Germany
}




\title{
A SHIELD-DATA-BASED HORIZON CONTROL APPROACH FOR THIN SEAM COAL MINING UTILIZING PLOW TECHNOLOGY
}

\begin{abstract}
The availability of renewable energy does not meet the demands of consumption. Hence, fossil fuels will continue to be an important energy source, and coal promises to cover the requirements for the coming decades. Because of its high energy content, deep lying bituminous coal and anthracite are important for energy generation. One trend in the future of coal mining is thin seam mining, because thick seams more and more have been exploited. A well-known and approved longwall mining method for thin seams is plowing which has big advantages compared to a shearer because of its compact design. The extraction of those thinner coal seams will become more and more important in coal industry and therefore the plow will receive more attention in future. Most approaches in longwall automation are focused on the shearer and need a more or less intelligent exploitation machine. Therefore those approaches cannot be used for the plow technology. The authors introduce a shield-data-based horizon control (SDHC). The developed approach integrates the shield data provided by the roof support and process monitoring into the horizon control process to keep the plow in seam even if the seam trend changes. This control concept can be used for the shearer as well as for the plow. The basic idea of SDHC for plow application will be introduced in this paper and first simulation results will be shown.
\end{abstract}

\section{KEYWORDS}

Mining, longwall, coal, thin seam, plow, horizon control, SDHC, automation

\section{INTORDUCTION}

The relevance of coal for future energy supply is undoubted. Big advantages in mining technologies and automation have increase the productivity and safety in underground coal industry. Because thick seams are more and more exhausted, mining in seams of medium and thin thickness will be more important. A well-known technology for thin seam mining is the plowing. In the 1st section of this paper a short introduction to the importance and automation standards of this technology is given. The 2 nd section introduces the horizon control. The automated horizon control is a control process for keeping the workface in the seam automatically and was developed for shearer technology in recent years. In the following section the authors present a new developed shield-data-based horizon control approach. Its principle ability of application to plow systems and the necessary data preparation are illustrated. The section Simulation Results shows the outcomes of a simulated test scenario. Finally the paper closes with a conclusion. 


\section{THIN SEAM MINING AND PLOW TECHNOLOGY}

For longwall mining two different exploitation methods exist, the shearer and the plow. Both machines are guided along the coal by the armored face conveyer (AFC) and the roof is supported by shields. The decision which of these systems is suitable for a particular deposit depends on numerous influences. Those influences are e.g. coal hardness, roof and floor conditions, face inclination, undulations, expected disturbances and seam thickness (Myszkowski \& Paschedag 2008; Scheidat \& Schwolow 1992). For thick seams the shearer is the method of choice. The shearer therefore was the point of interest for developments in automation in recent years; a short overview is given in (Beitler, Holm, Mozar, Junker, \& Bohn 2013). In thin seams the plow has big advantages due to its compact design. The plow technology is well known and looks back on a long history in European mining. A historical review is given in (Paschedag 2011). For medium seams there is a range, determined between $1.8 \mathrm{~m}$ and $2.3 \mathrm{~m}$ (Myszkowski \& Paschedag 2008), in which both systems could be used and the choice depends on other conditions as mentioned above. In the German Ruhr District more than $70 \%$ of coal is placed in seams thinner than $1.8 \mathrm{~m}$ (Daul \& Juch 1999). So it is not surprising that the plow was the dominating exploitation machine in German mining with a percentage of $74 \%$ in the early 1990s (Scheidat \& Schwolow 1992). The importance of thin seam mining is also present in U.S. thin seam mining (Weißdack \& Kvitkovich 2006) and even Chinese mining companies increase their number of automated plow systems (Tang 2011).

Nowadays the plow and the relevant equipment in a modern workface is a high automated and very productive system. Plow workfaces have reached a high degree of automation due to its application field in thin seams that sometimes does not allow an operator to stay in the workface during operation. Therefore the operator controls the exploitation process from a central control station in underground or even at the surface by remote control. Subordinated automations coordinate the movement of the AFC and the roof supports. High performance plow systems are able to push the AFC a predefined way (the next cutting depth) and generate a predefined workface. Furthermore modern systems like the CAT automated plows provides a vertical horizon control by utilizing the outrigger steering system. This system provides a downward or upward cutting direction by activating a hydraulic cylinder between AFC and the relay bar of the roof support to keep the workface in seam. In the best of the authors' knowledge, there exist no control systems that specify the horizon for the plow to follow the coal seam. Until today, this is the task of an operator.

\section{HORIZON CONTROL FOR FULLY AUTOMATED LONGWALL MINING}

Developments in the field of longwall automation were often focused on the shearer in the past. The shearer was declared as the key equipment for reaching fully automation (Wang, Ni, Li \& Xu 2009). A widely accepted automation modus is the so-called memory cut, whereby the shearer repeats a before taught path. But not only the path, also cutting drum height adjustment, speeds and other parameters can be repeated assigned to the shearer position. The memory cut can achieve good results as long as the seam has a continuous and smooth shape. The memory cut is not a full automated system. The mining process still needs the monitoring of an operator. 
A lot of research is focused on control strategies to extend the memory cut by utilizing an automated height adjustment for cutting drums to achieving horizon control. Classical strategies seem to be inadequate because of the unpredictable system behavior and so work often is attached to biological inspired methods or to neuronal networks (Beitler, Holm, Mozar, Junker, \& Bohn 2013). Another memory cut based automation approach is the enhanced horizon control (EHC) (Reid, Henderson, Hainsworth, Roberts, Chako, McLeod \& Mehl 2005) as an outcome of the ARCAP landmark project. The EHC generates roof and floor offsets out of different information like inertial navigation system (INS), coal interface detection (CID), a seam model and others. These offsets revise the calculated height from the OEM memory cut controller. The EHC and the other above mentioned approaches are focused on the shearer and use its "intelligence" to achieve automated horizon control and therefore they are not transferable to the plow technology. In cooperation with the German mining company RAG Deutsche Steinkohle a different approach in automation of longwall coal mining was developed, the Shield-Data-Based Horizon Control (SDHC). This concept is applicable for shearer and plow longwall mining.

\section{SHIELD-DATA-BASED HORIZON CONTROL (SDHC)}

\section{Principle Of The SDHC And Its Application To Automated Plow Systems}

As its name applies, the SDHC requires the shield data to calculate the necessary parameters for the next cut and so follows the coal seam. The shield data therefore are stored to a data base, and provided to further analysis and preparation. The principle of the shield data analysis for Longwall mining with a plow is given in (Junker \& Mozar 2010). The edited data is subsequently provided to the SDHC control algorithm. After calculation, the parameters are transmitted to the final controlling equipment, which can be the shearer and its cutting drum height adjustment or the outrigger steering of the AFC for plow application.

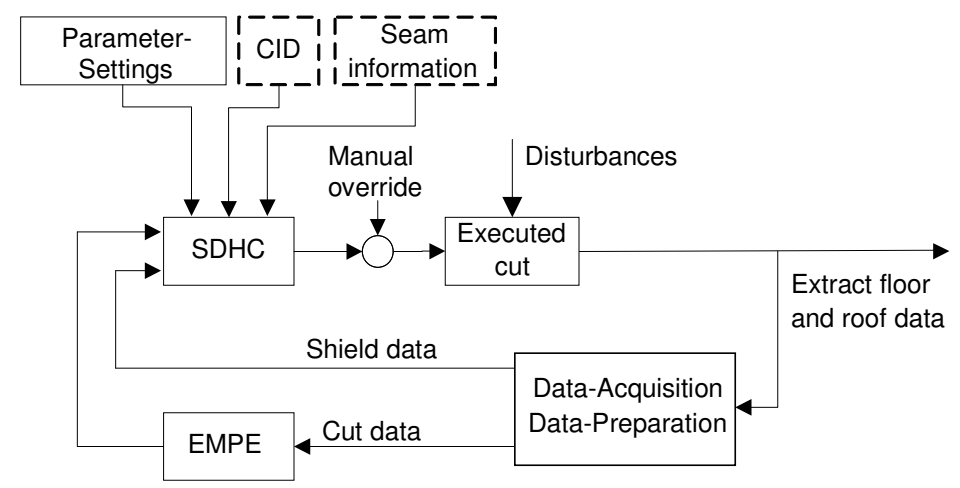

Figure 1 - Block diagram of the SDHC control loop

The basic structure of the SDHC control loop is shown in Figure 1. This block diagram applies for shearer and plow faces similarly. It can be seen, that the main inputs of the SDHC, beside the parameter settings, are the already mentioned edited shield data and the output data from the exploitation machine positioning estimator (EMPE). The EMPE provides an estimated position of the AFC (and thus of the cutting machine) for the next run based on shield data, pre- 
vious position, cut work and geometrical relations. The principle of a shearer positioning estimation is given in (Xu \& Wang 2010) and can be transferred to geometrical relations and the behavior of a plow system.

The task of the SDHC controller is to recognize the seam trend by analyzing the shield data and to follow this trend with a nearly constant height of roof supports. One requirement is that coal above the executed cut is parting at a separating layer between coal and rock or nearby. It is assumed that the roof represents the seam trend and therefore it is the command variable. Hence, the second task of the SDHC is to avoid cutting into the roof. By cutting into the roof shield data would present an environment, which does not follow the natural trend of the seam and the SDHC would probably fail its main task. As indicated by the dashed blocks, the SDHC does not need CID or offline seam information, but could be improved by taking that information into account. Compared to other approaches, which are focused on the cutting machine, the SDHC takes information from all involved components into account. Therefor the SDHC has to be implemented in a superordinate control level of the mine monitoring system.

The SDHC for automated plow systems is a modification of the SDHC developed for the shearer before. In consequence of this modification, various adaptations have to be done, e.g. the EMPE like already mentioned above. Also the SDHC control algorithm itself has to be adapted because the shearer requires height adjustments for the cutting drums while outrigger steering requires an angle to adjust. Furthermore the data acquisition and data preparation requires more attention when utilizing a plow; this will be outlined in the following.

\section{Data Acquisition And Data Preparation}

In the case of utilizing a shearer, the cutting drum height can be adjusted at every single position along the face by the shearer; and every position is related to a specific shield. Thus, the SDHC can calculate the next height at a specific position from directly corresponding shield data. These data are provided to the SDHC in form of an input vector, consisting of different heights, angles, and distances. Each vector corresponds to one shield. In comparison to this, the outrigger steering of a plow system is not implemented at every pan and so the inclination cannot be adjusted at every position. The outrigger steering is typically implemented in every sixth shield and so an inclination adjustment has to consider all shields in a block and its neighbor also.

The first step in data acquisition is a time-based database for all relevant workface parameters to be stored. Such a data base is commonly installed in modern faces for monitoring applications. Data from this database are transferred into a second one, where all data are associated to a specific face advance. From this "cutting path oriented" database a parameter matrix $\boldsymbol{P}^{m \times n}$ is extracted which contains all necessary data for the SDHC from one shield block. The dimension of $\boldsymbol{P}$ is dependent to the number of parameters per shield $(n)$ and shield quantity per block $(m)$. In the next step, all these parameters have to be weighted corresponding to their relevance for the next adjustment. The evaluation of the relevance of specific parameters depends on different influences like trustworthiness and age of data. Also the distance between the outrigger steering unit and the sensor is important. So data from a shield near the coal face are regarded as 
more important for workface estimation than shields which are staying back. On the other hand, weighting factors of data from shields of block boundary have to take into account that an inclination adjustment at the steering unit also has to reach a reaction at the block boundary. The weighting factors are resumed in the weighting matrix $\boldsymbol{W}^{n \times m}$. By multiplying the weighting matrix and the parameter matrix, the resulting square matrix $\boldsymbol{R}^{n \times n}$

$$
\boldsymbol{R}=\boldsymbol{W} \cdot \boldsymbol{P}=\left[\begin{array}{cccc}
w_{\mathrm{p}, 11} & w_{\mathrm{p}, 21} & \ldots & w_{\mathrm{p}, m 1} \\
w_{\mathrm{p}, 12} & & & \\
\vdots & & \ddots & \\
w_{\mathrm{p}, 1 n} & & \ldots & w_{\mathrm{p}, m n}
\end{array}\right] \cdot\left[\begin{array}{cccc}
p_{11} & p_{12} & \ldots & p_{1 n} \\
p_{21} & & & \\
\vdots & & \ddots & \\
p_{m 1} & & \ldots & p_{m n}
\end{array}\right]
$$

contains all necessary weighted parameters for the SDHC input vector on its principal diagonal. So the input vector is defined as

$$
\boldsymbol{f}=\left[\begin{array}{llll}
r_{11} & r_{22} & \ldots & r_{n n}
\end{array}\right]
$$

After this data preparation, the calculated input vector can be processed by the SDHC similarly to the shearer case. When utilizing the plow, the SDHC calculates an inclination adjustment for every block outrigger steering unit. This process of data acquisition, data preparation and calculation is done successively. After the plow has passed a shield, and the AFC has pushed toward the coal face, the EMPE estimates the new AFC position and the current shield data is stored into the database. This is done for every shield in the work face until a data set for a predefined block is available. After that, the data preparation is done automatically, the input vector is sent to the SDHC controller and the calculation starts immediately. The result is stored to a cuttemplate and provided to the outrigger steering for the next cut.

\section{SIMULATION RESULTS}

After the adaption of the EMPE to a real plow system utilized by the RAG Deutsche Steinkohle, the current work is focused on the data preparation and the implemented control algorithm of the SDHC. To design, test and optimize the controller a simulator of the RAG Deutsche Steinkohle is used. The simulator is developed to compute the cutting and moving behavior of a plow or shearer and one corresponding roof support in the seam. So the simulator provides data of one shield and the corresponding cut data. Thus, tests of the data preparation could not be realized with the actual simulator version. By using this simulator, the controller could be tested in different scenarios like seam inclination, undulations and changes in face heights along the seam.

A demonstration of one test scenario and its result visualization is shown in Figure 2. In this test, the seam has a constant height of $1.2 \mathrm{~m}$; the roof and floor are illustrated by grey lines. It can be seen, that the seam starts an upward trend at approximately $3 \mathrm{~m}$ face advance and gets back to a horizontal layer, $40 \mathrm{~cm}$ higher than it starts, at $14 \mathrm{~m}$ and stays in it. The task of the SDHC is to follow the upward trend autonomously and reach the horizontal part of the seam without cutting into the roof and keeping the seam height at a nearly constant level. The plow and 
the roof support (indicated by its important geometrics) have reached a face advance of $27 \mathrm{~m}$ in this simulation. The roof collapse in the goaf is not considered in the simulation.

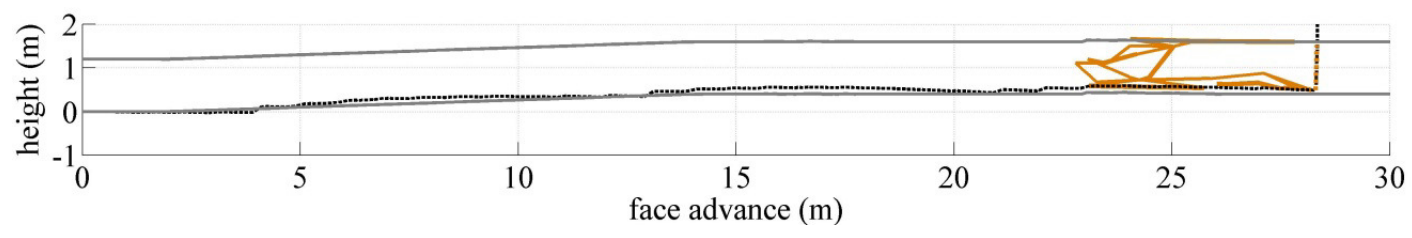

Figure 2 - Simulation result of the SDHC for a specific test scenario

The resulting floor profile is indicated by the dashed line. Because of the assumption, that coal above the executed cut parting at the coal rock interface, the resulting roof is equal to the given roof in simulation, except the case when cutting into the roof. If the roof is injured, it would be indicated by a dashed lined roof profile similar to the floor. So it can be seen, that the plow follows the seam automatically without injuring the roof. At a face advance of $3 \mathrm{~m}$, the upward trend of the seam starts. It is recognizable, that the plow starts its upward movement with a delay of approximately $1 \mathrm{~m}$, with a small overshoot which leads to a decrease in face height. After a transient, the plow is back in seam and reached the desired height at approximately $10 \mathrm{~m}$. A similar phenomenon occurs at $14 \mathrm{~m}$, where the seam turns back into the horizontal situation. The delay leads to a decreasing seam height, then the plow starts his adjustment and the plow system recreate the face height at approximately $20 \mathrm{~m}$ and try to align the system to the seam in the further face advance.

The delay of adjustment is attributable to the circumstance, that the roof support passes a face advance position later than the cutting machine. Therefore an adjustment can be activated as recently as changes in the seam can be identified in shield data. The delay of approximately $1 \mathrm{~m}$ is acceptable even in comparison with a human operator. The big advantage of the SDHC is the opportunity to recognize changes along the whole face in every step and therefore provide autonomously horizon control.

\section{CONCLUSION}

Thin seam longwall mining will become more and more important in future coal mining and therefore the plow technology will receive more attention. A modern plow workface delivers a high demand on automation including a horizon control by outrigger steering. Until today the trend of a seam has to be evaluated by an operator and the automation degree is restricted. To overcome those restrictions the SDHC for plow systems is introduced. The SDHC uses shield data and cut data to evaluate seam trends and utilizes the outrigger steering unit for automated horizon control. Simulation results have shown that the controlled system is enabled to follow a seam trend autonomously and allow a new view on a higher automation degree in longwall mining with the plow technology. Regarding the promising simulation results of the developed SDHC controller, the next step is to optimize the controller parameters and the data preparation. 
Additionally, special attention will be given to its implementation into a monitoring system to prepare first real scenario tests in cooperation with the RAG Deutsche Steinkohle.

\section{ACKNOWLEDGEMENT}

For the support during the development and writing process the authors wish to thank E. Clausen for sharing her expertise and time to give information about the underground mining process. The authors also thank the employees of the Otto v. Guericke University of Magdeburg,

C. Daniel and E. Woschke for implementing the workface behavior into the simulation model.

\section{REFERENCES}

Beitler S., Holm M., Mozar A., Junker M. \& Bohn C. (2013). State Of The Art In Underground Coal Mining Automation and Introduction of a New Shield-Data-Based Horizon Control Approach. Accepted for publication. $13^{\text {th }}$ International Multidisciplinary Scientific GeoConferences SGEM. Albena, Bulgaria, June 2013.

Daul J. \& Juch D. (1999). Die Verteilung der Vorräte der Steinkohlenlagerstätten an der Ruhr vor dem Abbau und am 1. Januar 1995 (The distribution of reserves of hard coal deposits in the Ruhr District before exploitation and on $1^{\text {st }}$ of January 1995). Glückauf, Vol. 135(1/2). 34-42.

Junker M. \& Mozar A. (2010). Verfahren zur automatischen Herstellung einer definierten Streböffnung in Hobelbetrieben des Steinkohlenbergbaus (Method for automatic production of defined face opening in plow operation in coal mining), Patent application: DE 102008053133 A1 2012.05.06

Myszkowski M. \& Paschedag U. (2008). Longwall mining in seam of medium thickness - comparisons of plow and shearer performance under compareable conditions. Lünen, Germany: Bucyres.

Paschedag U. (2011). Plow technology - history and today's state-of-the-art. Proceedings of the International Mining forum 2011. Bogdanka, Poland, November 2011. 1-15.

Reid D., Henderson P., Hainsworth D., Roberts B., Chacko A., McLeod C. \& Mehl R. (2005). Interconnection of Landmark Compliant Longwall Mining Equipment - Shearer communication and functional specification for enhanced horizon control. (CSIRO Exploration and Mining Report P2004/6, Version 2.36). Retrieved from: http://www.lascautomation.com/docs/HorizonControlSpecificationV2.36.pdf $\quad$ (15. October 2012)

Scheidat L. \& Schwolow G. (1992). Schälende oder schneidende Gewinnung in Flözen mittlerer Mächtigkeit. (Stripping or cutting extraction of seams of medium thikness). Glückauf, Vol. 128(1). 53-54.

Tang D. X. (2011). Operational experiences of automated plow systems in Tiefa, China. Proceedings of the International Mining forum 2011. Bogdanka, Poland, November 2011. 29-35.

Wang Z., Ni W., Li S. \& Xu Z. (2009). Research on key technologies of remote monitoring platform for shearer. Proceedings of the IEEE International Conference on Measurement Technology and Mechatronics Automation. Zhangjiajie, Hunan, China, April 2009. Vol. 1. 316-319.

Weißdack G. V. \& Kvitkovich J. F. (2006). U.S. longwall development projected to increase. Mining Engineering. Vol. 58(1). 27-32.

Xu Z. \& Z. Wang. (2010). Research on key technology of shearer position. Proceedings of the IEEE International Conference of Intelligent System Design an Engineering Application. Changsha, Hunan, China, October 2010. 280-283. 